\title{
CONF-970629--3
}

\section{THERMODYNAMICS AND KINETICS OF ION SPECIATION IN SUPERCRITICAL AQUEOUS SOLUTIONS: \\ A MOLECULAR BASED STUDY}

by

A. A. Chialvo ${ }^{12}$, P. T. Cummings ${ }^{1,2}$, J.M. Simonson ${ }^{3}$, and R.E. Mesmer ${ }^{3}$

${ }^{1}$ Department of Chemical Engineering, 419 Dougherty Engineering Bldg. University of Tennessee Knoxville, TN 37996-2200, U.S.A.

${ }^{2}$ Chemical Technology Division. Oak Ridge National Laboratory. Oak Ridge, TN 37831 6268, U.S.A.

${ }^{3}$ Chemical and Analytical Science Division. Oak Ridge National Laboratory. Oak Ridge, TN 37831-6110, U.S.A.

SUBMITTED FOR PUBLICATION TO: FLUID PHASE EQUIIIBRIA

May 1, 1997

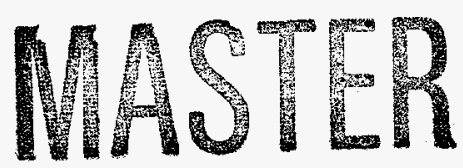

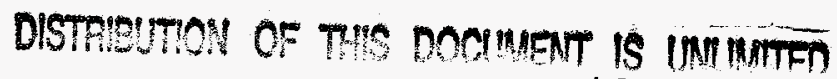

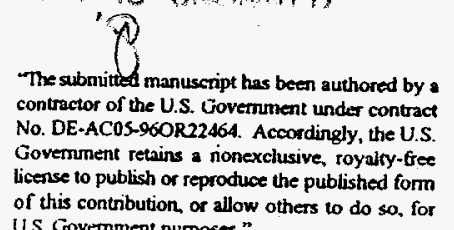




\section{DISCLAIMER}

This report was prepared as an account of work sponsored by an agency of the United States Government. Neither the United States Government nor any agency thereof, nor any of their employees, make any warranty, express or implied, or assumes any legal liability or responsibility for the accuracy, completeness, or usefulness of any information, apparatus, product, or process disclosed, or represents that its use would not infringe privately owned rights. Reference herein to any specific commercial product, process, or service by trade name, trademark, manufacturer, or otherwise does not necessarily constitute or imply its endorsement, recommendation, or favoring by the United States Government or any agency thereof. The views and opinions of authors expressed herein do not necessarily state or reflect those of the United States Government or any agency thereof. 


\section{DISCLAMMER}

Portions of this document may be illegible in electronic image products. Images are produced from the best available original document. 


\section{ABSTRACT}

Molecular simulation of infinitely dilute $\mathrm{NaCl}$ aqueous solutions are performed to study the $\mathrm{Na}^{+} / \mathrm{Cl}^{-}$ion pairing in a polarizable and a non-polarizable solvent at supercritical conditions. The Simple Point Charge, the Pettitt-Rossky, and the Fumi-Tosi models for the water-water, the ion-water, and the ion-ion interactions are used in the determination of the degree of dissociation, its temperature and density dependence, and the kinetics of the interconversion between ion-pair configurations in a non-polarizable medium. To assess the effect of the solvent polarizability on the stability of the ion-pair configurations we replace the Simple Point Charge by the Polarizable Point Charge water model and determine the anioncation potential of mean force at $T_{r}=1.20$ and $\rho_{r}=1.5$.

\section{INTRODUCTION}

Over the last few years we have been performing molecular-based studies of water and aqueous solutions to understand the microscopic mechanism of solvation, in hightemperature electrolyte and non-electrolyte solutions, through a combination of theory, simulation, and experiment ${ }^{1-7}$. High-temperature aqueous-electrolyte solutions are becoming the focus of renewed interest because of the significant role played by ion solvation in processes involving new environmentally-friendly technologies - such as the hydrothermal oxidation of toxic wastes ${ }^{8}$ - and well known complications with current technologies - such as metal corrosion and solid deposition in chemical reactors and steam power generators ${ }^{9}$.

Unlike ion solvation at ambient conditions, where the water dielectric screening tends to stabilize charged species, ionic species exhibit low solubility and speciation in low dielectric environments (such as those at supercritical conditions) ${ }^{10}$. This behavior introduces yet another complication to the modeling of aqueous electrolytes, and points to the need for a more fundamental (molecular-based) analysis of the solvation phenomenon. 
In an effort to shed some light into the high-temperature ion solvation, we have recently undertaken a rather extensive investigation on the temperature and density effects on the degree of ion association in supercritical aqueous $\mathrm{NaCl}$ solutions, through the molecularbased determination of the association ${ }^{6,11,12}$. In a first stage of this investigation, we proposed and discussed a methodology to study the ion-pair association in hightemperature dilute aqueous solutions. Then, we analyzed the reliability of several ion-water models in predicting the association constant, and in doing so, we argued that an important source of ambiguity in the evaluation of the constant of association is the reference value for the potential of mean force (integration constant). This integration constant is usually approximated by the (mean field) dielectric attenuated ion-pair Coulombic potential at a finite distance, at which we can assume that the solvent behaves as a dielectric continuum 13.14 .

Among other relevant conclusions, our previous simulation results highlighted the importance of an accurate description of the solvent's dielectric constant in the molecular simulation of aqueous electrolyte solutions, especially when dealing with mean-force calculations. In addition, those simulation results gave additional support to earlier claims about the reliability of the SPC water model, in particular, about the role played by the fortuitous compensation of factors (such as the under-prediction of the dielectric constant and the actual temperature, for a given corresponding state) involved in the integration constant of the potential of mean force (see Eq. (7) in Ref.11).

An important shortcoming of any non-polarizable water model (including the SPC model used in our previous studies) is its inability to describe the actual polarization anisotropy near charged species ${ }^{15}$, a feature that prevents a more realistic representation of the restructuring of water around the ions, i.e., the correct short-range polarization behavior. This becomes a serious defect when dealing with ion-pair solvation, since an ion pair forms a rather large dipole which polarizes the surrounding solvent, whose effect on the stability of the ion pair is neither obvious nor known. Thus, while the solvent dielectric 
plays a key role in the ion solvation, there are less obvious local effects associated with the solvent's polarizability which can influence the stability of the solvated species.

In this paper we present a first step toward the understanding of the solvent polarizability effect on high-temperature ion association. With this purpose in mind, we perform potential of mean force calculations for an infinitely dilute aqueous $\mathrm{NaCl}$ solution using the Polarizable Point Charge (PPC) ${ }^{16}$ (as opposed to the Simple Point Charge $(\mathrm{SPC})$ ), the Pettitt-Rossky ${ }^{17}$, and the Fumi-Tosi ${ }^{18}$ models for the water-water, the ionwater, and the ion-ion interactions at $T_{r}=1.20$ and $\rho_{r}=1.5$. The simulation results are compared with the corresponding in which the solvent is described by the SPC water model, to determine the extent of the solvent's polarizability effects on the stability of ionpair configurations.

The paper is organized as follows. In section 2 we first briefly review the main issues behind the molecular-based study of ion-pair association, and the kinetics of ion-pair interconversion in non-polarizable dilute aqueous (SPC) solutions. Then, we introduce the PPC water model and some details on its molecular dynamics implementation to study the polarizability effects on the anion-cation potential of mean force in a polarizable medium. In section 3, we discuss previous results for the non-polarizable SPC model to make contact with new simulation results corresponding to the polarizable PPC water model, and summarize some relevant conclusions.

\section{POTENTIAL OF MEAN FORCE IN A NON-POLARIZABLE AND A POLARIZABLE AQUEOUS MEDIUM}

\subsection{Potential of mean force and ion-pair association}

The association process between a pair free ions, $\mathrm{Cr}$ and $\mathrm{Na}^{+}$, to form a neutral associated pair $\mathrm{Na}^{+} \mathrm{Cl}^{-}$, can be envisioned as follows,

$$
\begin{aligned}
& \mathrm{Na}^{+}+\mathrm{Cl} \Leftrightarrow \mathrm{Na}^{+} \mathrm{Cl}^{-} \Leftrightarrow \mathrm{Na}^{+} \mid \mathrm{Cl} \Leftrightarrow \mathrm{Na}^{+} \| \mathrm{Cl} \\
& \text { free ions } \quad \text { CIP SShIP SSIP }
\end{aligned}
$$


where SSIP, SSHIP, and CIP stand for solvent-separated, solvent-shared, and contact ionpairs configurations, respectively ${ }^{19}$.

A typical molecular approach to study this association scheme hinges around the calculation of the potential of mean force $W(r)$ along the "reaction coordinate", e.g., the anion-cation separation. The potential of mean force represents the isothermal work done on the aqueous system to bring the anion and cation to a particular distance (pair configuration) in the presence of the solvent, and is directly connected to the system's microstructure for the anion-cation interactions (the radial distribution function $g_{N \alpha C l}(r)$ ) by $W(r)=-k_{B} T \ln g_{\mathrm{NaCl}}(r)^{20}$. Even though the microstructure is typically a by-product of any standard simulation, the condition of infinite dilution prevent us from determining $W(r)$ directly from the corresponding $g_{\mathrm{NaCl}}(r)$. Therefore, we must resort to a lengthy and non trivial methodology ${ }^{21.22}$ to evaluate $W(r)$ through a series of simulations as discussed in some detail elsewhere ${ }^{6}$.

\subsection{Kinetics of ion-pair interconversion}

Ion-pair interconversion is a relevant kinetic mechanism in inorganic ionic reactions

${ }^{23}$, i.e., the conversion process between the CIP and the SShIP/SSIP configurations as indicatod below,

$$
\mathrm{Na}^{+} \mathrm{Cl}^{-} \stackrel{\mathrm{k}_{f}}{\underset{\mathrm{k}_{b}}{\longrightarrow}} \mathrm{Na}
$$

CIP SShIP

where $k_{\mathrm{f}}$ and $k_{\mathrm{b}}$ are the corresponding dissociation and association kinetic rate constants. The process can be viewed as the recrossing of a free energy barrier between the CIP and SShIP configurations, and therefore, can be analyzed by the formalism for chemical reactions in solutions ${ }^{24}$. In doing so we can distinguish the reactant (CIP) from the product (SShIP) according to their position along the "reaction coordinate" relative to the 
location of the first maximum of the potential of mean-force $W(r)$, i.e., the free energy barrier.

According to the Transition State Theory (TST) ${ }^{25}$, the kinetic rate of dissociation (forward) of a CIP $k_{f}^{\text {TST }}$ (for $k_{\mathrm{f}}$ ) is directly connected to the anion-cation potential of meanforce as,

$$
k_{f}^{T T}=\frac{\left(r^{*}\right)^{2} \exp \left[-W\left(r^{\ddagger}\right) / k_{B} T\right]}{\left(2 \pi \mu_{\varphi} / k_{B} T\right)^{05} \int_{0}^{r^{*}} \exp \left[-W(r) / k_{B} T\right] r^{2} d r}
$$

where $k_{B}$ is the Boltzmann constant, $\mu_{i p} \equiv\left(m_{C l^{-}} m_{\mathrm{Na}^{+}} /\left(m_{\mathrm{Cl}^{-}}+m_{\mathrm{Na}^{+}}\right)\right)$is the reduced mass of the ion-pair, and superscript $¥$ denotes the location of the transition state or energy barrier between the CIP and SShIP. Similarly, the TST kinetic rate constant for the association process (backward) is given by,

$$
k_{b}^{T T}=\frac{\left(r^{t}\right)^{2} \exp \left[-W\left(r^{*}\right) / k_{B} T\right]}{\left(2 \pi \mu_{i p} / k_{B} T\right)^{0.5} \int_{F}^{r_{2}} \exp \left[-W(r) / k_{B} T\right] r^{2} d r}
$$

where $r_{2}$ indicates the location of the second local maximum of the $W(r)$ (corresponding to the transition between the SShIP and the SSIP.

Note that, in contrast to other types of reaction studied by simulation, ${ }^{26,27}$ which are characterized by a free energy barrier even in the absence of selvent, the ion-pair interconversion becomes possible due to the presence of the solvent. That is, the interconversion equilibrium owes its existence to the presence of the solvent, whose dielectric screening of the ion charges creates an energetic barrier resulting from the average ion-solvent interactions. This feature has important implications on the deviation of the observed kinetic behavior from the TST predictions as discussed in some detail elsewhere 12.13 .28 . 


\subsection{Solvent polarization effects}

To make a meaningful comparison with our earlier mean-force calculations based on the non-polarizable SPC water model ${ }^{29}$, and assess the solvent polarization effect, we use the Pettitt-Rossky model ${ }^{17}$ for the water-ion interactions, and the Fumi-Tosi model ${ }^{18}$ for the anion-cation interactions. The PPC water model is an attractive choice over other models because it appears to predict accurately the dielectric constant of water over a wide range of conditions.

The geometry of this model is similar to the TIP4P water model ${ }^{30}$, in that the negative charges are initially located in the bisectrix of the $\mathrm{H}-\mathrm{O}-\mathrm{H}$ angle (zero electric field), even though they are allowed to move in the molecular plane, and change their magnitude (constrained by the molecule's electroneutrality) in response to the local electric field (charge polarization). The rigid angle $H-O-H$ is $106^{\circ}$ and the $H-O$ bond length is $0.943 \AA$, i.e., $1.5^{\circ}$ larger and $0.014 \AA$ shorter than the corresponding values for the TIP4P model. As far as we are aware, this is the first mixture simulation based on PPC model.

All simulation were performed in the isokinetic-isochoric ensemble, with $N=254$ water molecules and the ion pair. Isothermal conditions were achieved by a Gaussian thermostat. The Newton-Euler equations of motion were integrated by Gear's 4 th order predictor -corrector ${ }^{31}$, and the rotational degrees of freedom were handled by Evans-Murad quaternion formalism ${ }^{32}$. At every time step the location and magnitude of the water electrostatic charges are determine to self consistency, by an iteration process starting with the prediction of the electric field at the oxygen (negative) site via a second order predictor. In average, the process takes typically two iterations to obtain self-consistent electrostatic fields, i.e., when the maximum change in the magnitude of the electric field at any site satisfies the criterion of convergency given by $\left(\delta E_{i}\right)^{2} /\left\langle E_{i}^{2}\right\rangle<0.0003$. 
The anion-cation mean force was determined by the constraint technique developed by Ciccotti et al. ${ }^{14}$, and the corresponding potential of mean force, by the usual integration and the mean field approximation for the constant of integration ${ }^{13}$. The production runs, a total of 20-22, for the mean force calculations comprised $2 \times 10^{5}$ time steps (approximately $200 \mathrm{ps)}$ for each anion-cation constrained distance. For the dielectric constant of the PPC water model, the run was extended to 500 ps. Additional simulation details are given in Refs. 6 and 11.

\section{SIMULATION RESULTS AND DISCUSSION}

\subsection{Temperature and density effects on ion-pair association}

Based on the formalism we developed ${ }^{6}$, we determined the degree of dissociation along the near-critical isotherm $T_{r}=1.05$, and along the supercritical isochore $\rho_{r}=1.5$ as shown in Figures 1 and 2. These simulation results indicated a temperature-density compensation of effects. In particular, they show a strong near-critical isothermal-density effect on the degree of dissociation of the ion pair (Figure 1), i.e., evidence of the solvent's dielectric screening of the ion charges. While the supercritical isochoric-temperature effect appears less pronounced than the near-critical isothermal-density effect, in both cases the simulation results predicted a rather strong dependence between the degree of dissociation and the total salt concentration (see Eqn. 5 in Ref. 11), a clear evidence that water at these supercritical conditions behaves as a mild solvation medium.

\subsection{Kinetics of ion-pair interconversion}

The main parameters for the determination of the rate constants along the reduced isotherm of $T_{r}=1.05$ are given in Table 1. Note that the energy barrier for the association and dissociation processes are approximately independent of the system's density. This 
feature, according to Eqns. (3)-(4), translates into kinetic rate constants with very weak density dependence as indicated in Table 2.

Table 1

Main features of the $\mathrm{Na}^{+}+\mathrm{Cl}^{-}$potential of mean force in SPC water $\left(T_{r}=1.05\right)$

\begin{tabular}{lccccc}
\hline $\begin{array}{l}\text { Reduced } \\
\text { density }\end{array}$ & $\begin{array}{c}\text { First } \\
\text { minimum }\end{array}$ & $\begin{array}{c}\text { First } \\
\text { maximum }\end{array}$ & $\begin{array}{c}\text { Second } \\
\text { minimum }\end{array}$ & $\begin{array}{c}\text { Dissociation } \\
\text { barrier }\left(\mathrm{k}_{\mathrm{B}} \mathrm{T}\right)\end{array}$ & $\begin{array}{c}\text { Association } \\
\text { barrier }\left(\mathrm{k}_{\mathrm{B}} \mathrm{T}\right)\end{array}$ \\
\hline 1.0 & $2.5 \AA$ & $3.9 \AA$ & $4.5 \AA$ & 3.55 & 0.40 \\
1.5 & $2.5 \AA$ & $3.9 \AA$ & $4.7 \AA$ & 3.66 & 0.44 \\
2.0 & $2.5 \AA$ & $3.9 \AA$ & $4.7 \AA$ & 3.57 & 0.31 \\
\hline
\end{tabular}

Table 2

TST rate constants (in $\mathrm{ps}^{-1}$ ) for the high-temperature ion-pair interconversion in SPC water $\left(T_{r}=1.05\right)$.

\begin{tabular}{ccc}
\hline$P / \rho_{c}$ & $k_{t}^{\text {TST }}$ & $k_{b}^{\text {TST }}$ \\
\hline 1.0 & $0.23 \pm 0.02$ & $0.57 \pm 0.01$ \\
1.5 & $0.24 \pm 0.02$ & $0.55 \pm 0.01$ \\
2.0 & $0.25 \pm 0.02$ & $0.44 \pm 0.01$ \\
\hline
\end{tabular}

According to the energy barrier of Table 1, and resulting TST rate constants, the association process SShIP $\rightarrow$ CIP should be more favorable than the dissociation process CIP $\rightarrow$ SShIP in the absence of any barrier recrossing. However, this recrossing does occur and its magnitude is given by the corresponding transmission coefficient. In fact, preliminary simulation results ${ }^{12}$, based on the time dependence of the reactive flux correlation function ${ }^{24}$, indicates that the transmission coefficient for the ion- 
pair interconversion at near-critical conditions is indeed very small, i.e., of the order of 0.05 .

\subsection{Solvent polarization effects}

Since the phase envelope for the PPC model is not available yet, and because this model predicts the dielectric constant and the second virial coefficient extremely accurately 16, the simulations were conducted at $T=776 \mathrm{~K}$ and $\rho=0.483 \mathrm{~g} / \mathrm{cc}$, which after assuming the critical conditions of real water, i.e., $T_{c}=647 \mathrm{~K}$ and $\rho_{c}=0.322 \mathrm{~g} / \mathrm{cc}^{33}$, represents $T_{r}=1.20$ and $\rho_{r}=1.5$. At these conditions, the PPC model predicts a dielectric constant of $8.0 \pm 0.2$ which compares very well with 8.1 from the correlation of Haar et al. ${ }^{33}$.

In Figures 3 and 4 we display the mean force and potential of mean force for the PPC model, compared with those for the SPC model. The most obvious feature in Figure 3 is that, for anion-cation distances $r_{\mathrm{NaCl}}>5 \AA$, the solvent contribution to the anion-cation interaction appears to be unaffected by the solvent polarizability. In other words, the solvent behaves as a continuum dielectric which attenuates the Coulombic charges on the ions. As the ions are pushed to shorter distances, i.e., $r_{\mathrm{NaCl}}<5 \dot{A}$, the increasing anisotropy of the electric field modifies the water charges unevenly (polarization in response to the local strength of the electric field), making an additional contribution to the anion-cation mean force. Note that this phenomenon is not taken into account in the case of non-polarizable water models such as SPC. In terms of potential of mean force, the solvent polarization at short anion-cation distances reverses the stability of the CIP and SShIP with respect to the observed behavior for the non-polarizable model (Figure 4), i.e., the second valley of the potential (corresponding to the SShIP configuration) is more negative than the first valley (corresponding to the CIP configuration). This behavior also suggests the need for explicit polarizable ion-ion and ion-water potentials. 


\section{FINAL REMARKS}

We have performed molecular simulations of aqueous electrolyte solutions, using intermolecular potentials taken from the literature, to study ion solvation at supercritical conditions. While our simulation results provide important insight into the mechanism of the ion-pair association, they also indicate the need for more accurate intermolecular potential models not only for water but also for the ions in solution. This is precisely one of the targets of our current research effort.

\section{ACKNOWLEDGEMENTS}

This work was supported by the Division of Chemical Sciences, Office of Basic Energy Sciences, U.S. Department of Energy. The work of JMS and REM was supported by the Division of Chemical Sciences, Office of Basic Energy Sciences, U. S. Department of Energy at Oak Ridge National Laboratory, managed Lockheed Martin Energy Re s carporation for the U.S. Department of Energy under contract DE-AC05-960R22464.

\section{CITED REFERENCES}

1 P. T. Cummings, H. D. Cochran, J. M. Simonson, R. E. Mesmer, and S. Karaborni, Journal of Chemical Physics 94, 5606-5621 (1991).

2 P. T. Cummings, A. A. Chialvo, and H. D. Cochran, Chemical Engineering Science 49, 2735-2748 (1994).

3 A. A. Chialvo, and P. T. Cummings, Journal of Chemical Physics 101, 4466-4469 (1994).

4 P.C. Ho, D.A. Palmer, and R.E. Mesmer, Journal of Solution Chemistry 23, $997-$ 1018 (1994).

5 A. A. Chialvo, P. T. Cummings, H. D. Cochran, J. M. Simonson, and R. E. Mesmer In Innovations in Supercritical Fluids: Science and Technology; K. W. Hutchenson and N. R. Foster, Ed.; American Chemical Society: Washington, D.C., 1995; Vol. 608; pp 65-76.

6 A. A. Chialvo, P. T. Cummings, H. D. Cochran, J. M. Simonson; and R. E. Mesmer, Journal of Chemical Physics 103, $9379-9387$ (1995). 
7 A. A. Chialvo, and P. T. Cummings, Journal of Physical Chemistry 100, 1309-1316 (1996).

8 H. E. Barner, C. Y. Huang, T. Johnson, G. Jacobs, M. A. Martch, and W. R. Killilea, Journal of Hazardous Materials 31, 1-17 (1992).

9 B. E. Conway, Chemical Society Reviews 253-261 (1992).

10 R. E. Mesmer, D. A. Palmer, and J. M. Simonson In Activity Coefficients in Electrolyte Solutions; 2nd Edition ed.; K. S. Pitzer, Ed.; CRC Press: Boca Raton, 1991; pp 491-529.

11 A. A. Chialvo, P. T. Cummings, J. M. Simonson, and R. E. Mesmer, Journal of Chemical Physics 105, 9248-9257 (1996).

12 A. A. Chialvo, P. T. Cummings, J. M. Simonson, and R. E. Mesmer, Journal of Molecular Liquids (1996).

13 E. Guàrdia, R. Rey, and J. A. Padró, Chemical Physics 155, 187-195 (1991).

14 G. Ciccotti, M. Ferrario, J. T. Hynes, and R. Kapral, Chemical Physics 129, 241251 (1989).

15 S.-B. Zhu, S. Singh, and G. W. Ribinson In Modern Nonlinear Optics; M. Evans and S. Kielich, Ed.; John Wiley \& Sons, Inc.: New York, 1994; Vol. 85; pp $627-$ 732.

16 I. M. Svishchev, P. G. Kusalik, J. Wang, and R. J. Boyd, Journal of Chemical Physics 105, 4742-4750 (1996).

17 B. M. Pettitt, and P. J. Rossky, Journal of Chemical Physics 84, 5836-5844 (1986).

18 M. P. Tosi, and F. G. Fumi, Joumal of Physics and Chemistry of Solids 25, 45-52 (1964):

19 M. Eigen, and K. Tamm, Z. Elektrochem. 66, 93-106 (1962).

20 D. A. McQuarrie Statistical Mechanics; Harper and Row, New York: 1976.

21 E. Guàrdia, A. Robinson, and J. A. Padró, Journal of Chemical Physics 99, 42294230 (1993).

22 G. Hummer, D. M. Soumpasis, and M. Neumann, Molecular Physics 77, 769-785 (1992).

23 R.M. Fuoss, Journal of Solution Chemsitry 7, 771-782 (1978)

24 J. T. Hynes In Theory of Chemical Reaction Dynamics; M. Baer, Ed.; CRC Press: Boca Raton, 1985; Vol. IV; pp 171-234.

25 P. Pechukas In Dynamics of Molecular Collisions; W. H. Miller, Ed.; Plenum Press: New York, 1976; Vol. B.

26 B. J. Gertner, K. R. Wilson, and J. T. Hynes, Journal of Chemical Physics 90 , 3537-3758 (1989). 
27 J. E. Straub, M. Borkovec, and B. J. Berne, Journal of Chemical Physics 89, 48334847 (1988).

28 D. E. Smith, and A. D. J. Haymet, Journal of Chemical Physics 96, 8450-8459 (1992).

29 H. J. C. Berendsen, J. P. M. Postma, W. F. van Gunsteren, and J. Hermans In Intermolecular Forces: Proceedings of the Fourteenth Jerusalem Symposium on Quantum Chemistry and Biochemistry; B. Pullman, Ed.; Reidel: Dordrecht, 1981; pp 331-342.

30 W. L. Jorgensen, J. Chem. Phys. 77, 4156 (1982).

31 C. W. Gear "The Numerical Integration of Ordinary Differential Equations of Various Orders," Argonne National Laboratory, 1966.

32 D. J. Evans, and S. Murad, Molecular Physics 34, 327-331 (1977).

33 L. Haar, J. S. Gallagher, and G. S. Kell Steam Tables; Hemisphere Publishing Corporation: New York, 1984. 
Figure 1

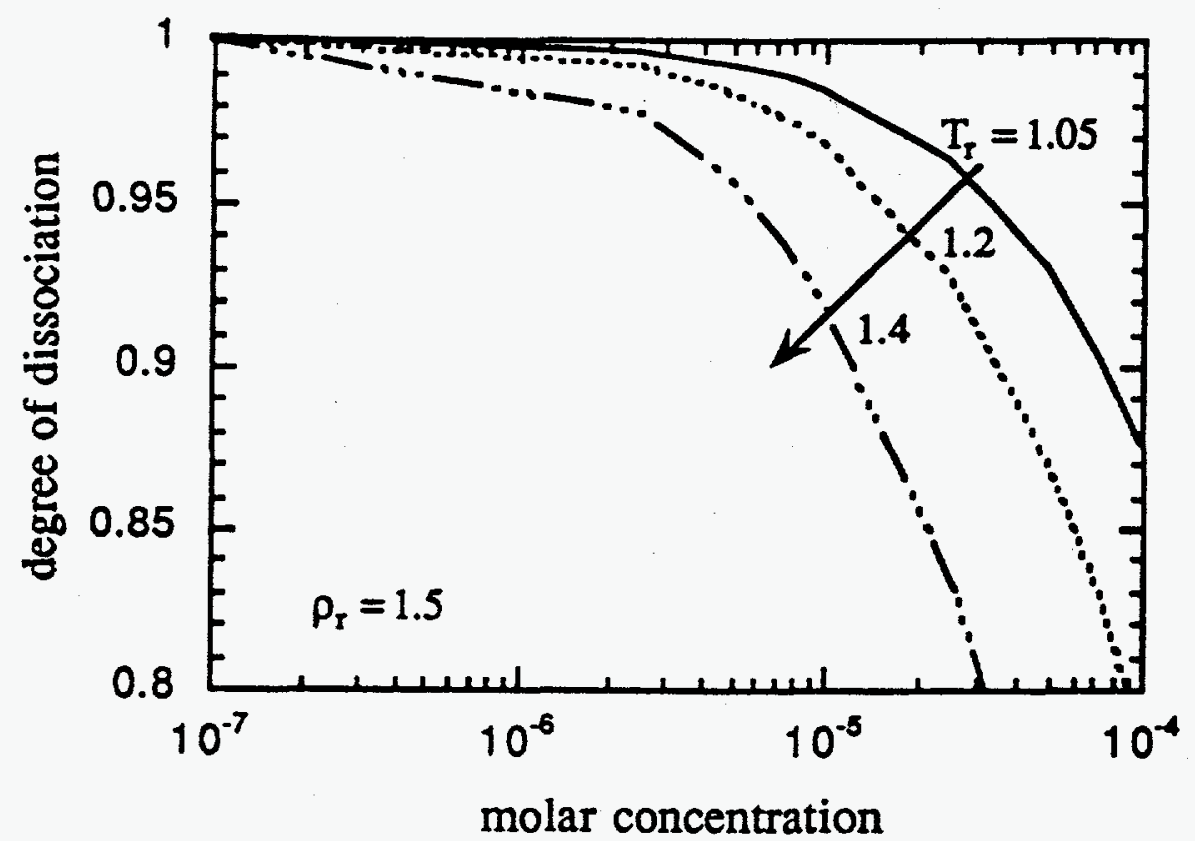

Figure 2

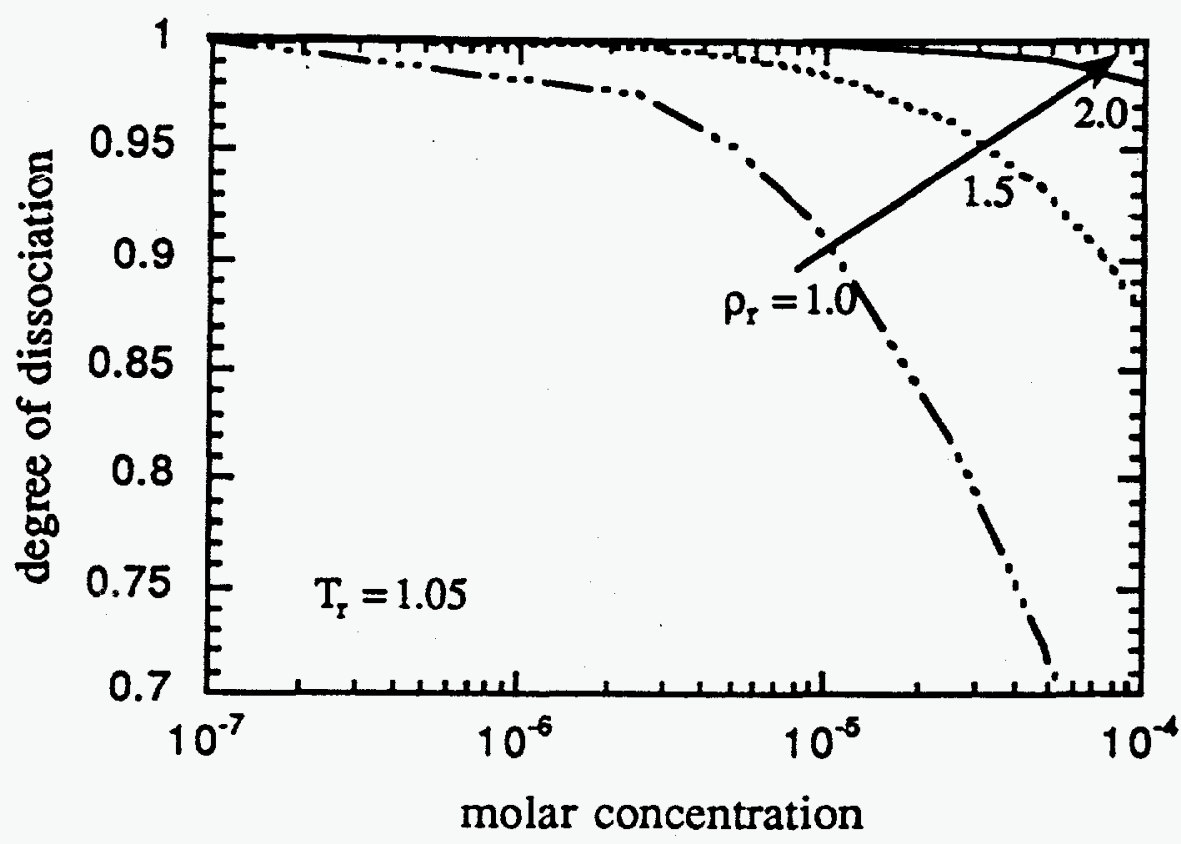


Figure 3

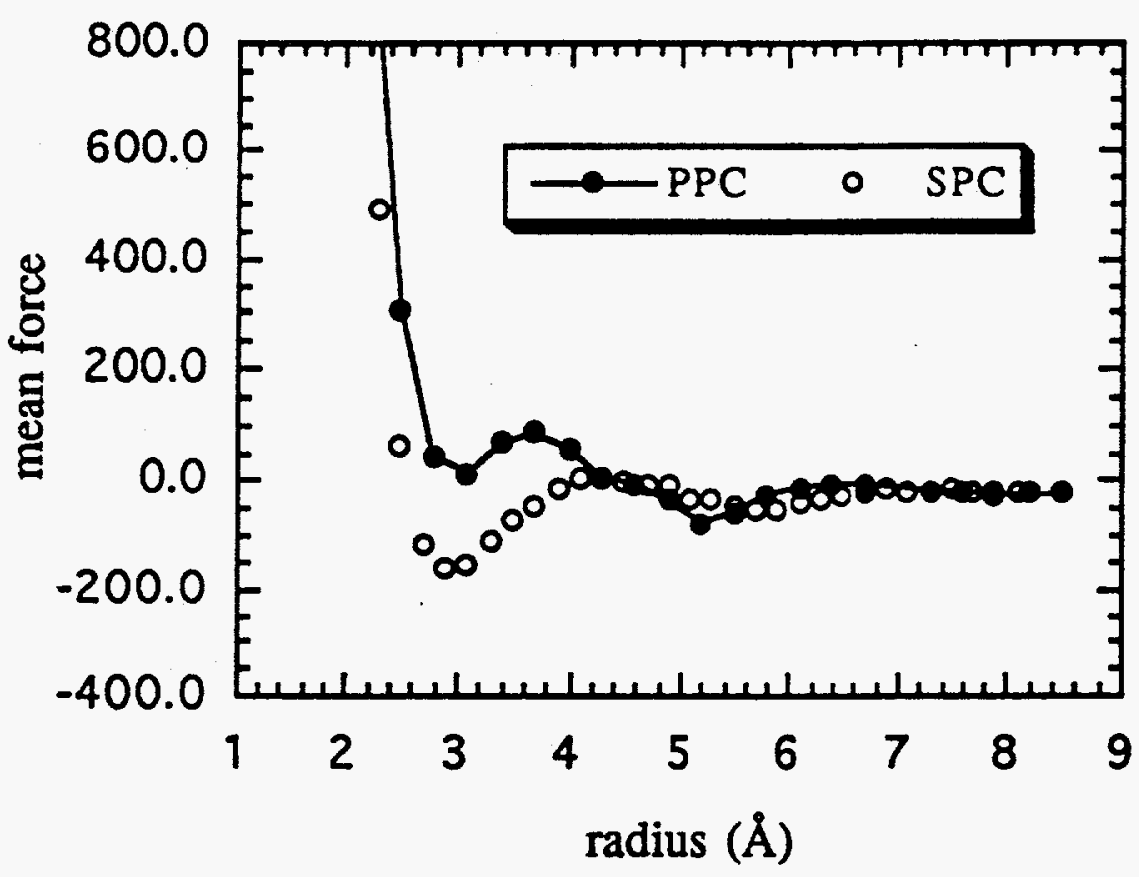

Figure 4

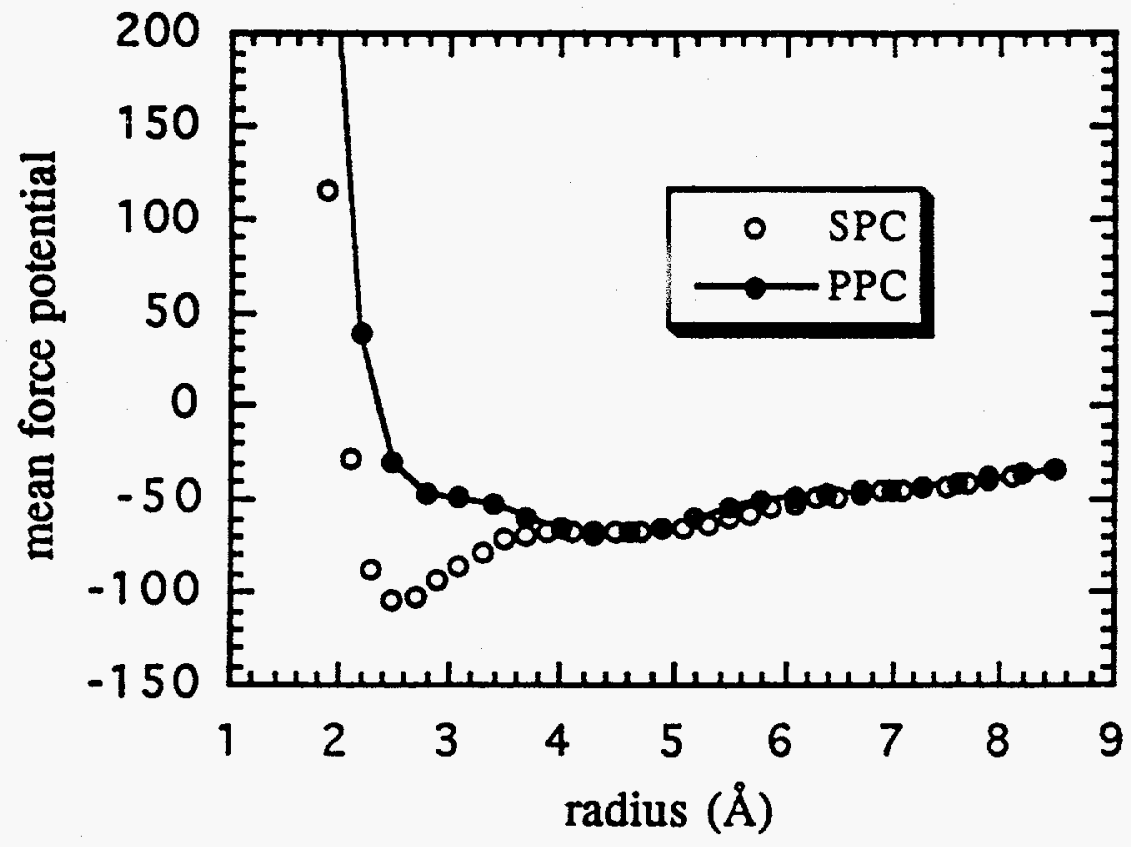

